\title{
MATERIAL PLACEMENT IN THE NEARSHORE: LABORATORY AND NUMERICAL MODEL INVESTIGATION
}

\author{
Bradley D. Johnson ${ }^{1}$ and Ernest R. Smith ${ }^{2}$
}

\begin{abstract}
Typical practice for a century has been to transport dredged sand to an offshore disposal site in deep water where the sediment is lost from the littoral system. The alternative of nearshore placement can retain the sand, but the fate of the material is poorly understood. A set of laboratory experiments were conducted, using tracer sand, with the intent of quantifying the migration of material with alternative dredged mound placements within the surf zone. Conventional depth-integrated tracer sand transport models can utilize a correction factor or a gradient diffusion mechanism to represent the effects of the depth variation. In the surf zone, however, an analytical correction factor is not available and a gradient diffusion coefficient is arbitrary with no physical basis. An alternative simple advective transport sand model is introduced herein that explicitly predicts both the advection associated with the return current and the wave-related onshore transport. With a simple framework based on a suspended layer and a bedload layer of arbitrary transport directions, the Taylor dispersion of tracer sand is explicitly computed without any dependence on a diffusion mechanism. Both the modeled and measured results indicate transport directed offshore by the undertow, onshore by the wave asymmetry, and down-drift as forced by the longshore current.
\end{abstract}

\section{INTRODUCTION}

Dredged material generated in the maintenance of navigable depths within channels is often comprised of high quality sand, especially along beaches with appreciable longshore sediment transport. The typical practice for a century has been to transport dredged sand to an offshore disposal site in deep water where the sediment is lost from the littoral system. The loss of this natural resource, obviously, can negatively impact the down-drift beach with a localized shoreline recession. The strategies for reducing dredging impacts include the nearshore placement of removed material inside the surf zone or in several meters of water depth, outside of the calm weather surf zone but within the littoral region. Wind waves, in theory, then act as the agent to winnow the fines and the facilitate onshore beach sand transport. While the benefits of preserving the sand and using waves for beach-face placement are obvious, there is no consensus on the effectiveness of this concept, especially considering the increased cost of nearshore deposition. Optimal placement and the particulars of the onshore sand migration, for instance, are largely unknown. Numerical modeling of mixing and transport may provide some insight and predictive capability in this balance. Indeed, nearshore morphology models predicting storm-scale erosion have been in use for the past several decades and demonstrate an acceptable level of accuracy. However, sediment transport models developed to predict cross-shore storm erosion are dominated by the seaward-directed wave and roller return current predicted in energetic seas. The onshore migration of sand and beach recovery is a gradual process and only prevails during period of low wave steepness (e.g. Dalrymple 1992), and the erosion models are poorly suited to longer-term simulations.

The focus of the present study is to introduce a conceptual model for the transport and mixing with a realistic representation of the advective process in the surf zone. The time-evolution of the placement sand is determined numerically, and the model remains computationally efficient with two coupled and vertically integrated layers. Comparisons of the model result with laboratory data are provided for the bed surface layer.

\section{LABORATORY STUDY}

A brief description of the Large-scale Sediment Transport Facility (LSTF) is given herein, and the interested reader is referred to the exhaustive report on the design, instrumentation, and capabilities of the tank by Hamilton et al. [2000]. The LSTF measures approximately $25 \mathrm{~m}$ in the cross-shore by $30 \mathrm{~m}$ alongshore where the walls are angled relative to a cross-shore transect to act as wave guides for the obliquely incident waves. Unidirectional waves were generated with four synchronized paddles

\footnotetext{
${ }^{1}$ US Army Engineer Research and Development Center (ERDC) 3909 Halls Ferry Rd, Vicksburg, MS 39180-619, Bradley.D.Johnson@erdc.usace.army.mil

${ }^{2}$ US Army Engineer Research and Development Center (ERDC) 3909 Halls Ferry Rd, Vicksburg, MS 39180-619, Ernest.R.Smith@erdc.usace.army.mil
} 
COASTAL ENGINEERING 2012

rotated to create long crests oriented $10^{\circ}$ from the long straight shoreline. A pump system was designed to re-circulate the wave-generated longshore current from the downstream boundary to the upstream boundary of the facility, thus allowing the laboratory basin to function as a infinitely long cylindrical coast. The cross-shore distribution of the longshore current is regulated by 20 independently controlled pumps and channels at the down-drift end of the tank. A schematic of the wave basin is provided in Figure 1, showing the inlet, outlet manifolds and the four wave paddles. The sand beach was comprised of well-sorted quartz sand with a median grain diameter of $0.15 \mathrm{~mm}$. Irregular waves were generated with a peak period of $T_{p}=1.5 \mathrm{~s}$ and a root-mean squared wave height of $0.12 \mathrm{~m}$. Basin currents and waves were measured with an array of instrumentation spanning the cross-shore transect of the LSTF. Instrumentation consisted of 10 wave gauges and 10 ADVS distributed in the cross-shore direction. The results from two configurations, one mid surf and one inner surf, are provided herein. To assure the use of sediment with the identical relevant material characteristics, basin sand was dyed to distinguish the tracer material from the native sediment The idealized mound constructions were comprised of $0.17 \mathrm{~m}^{3}$ of sand with a footprint of $2.4 \mathrm{~m}^{2}$ and positioned as shown in Figure 1. The mound in Test 1 was located in $-6.1<x<-5.4$ and $-29<y<-26$. Test 2 , in shallower water, was placed in $-3.64<x<-2.78$ and $-30<y<-26$.

A terrestrial LIDAR system was used to collect high resolution bottom position data. The optics-based method required draining the water from the basin, and bathymetry was collected after every ten minutes of wave action. In addition to location and elevations, the survey system included an image scanner, which collected red, green, and blue (RGB) components at each position on the sand bed. The dyed mound sand and the native sand color was differentiated through image processing. The tracer sand on the beach surface, then, was tracked at the times of bathymetry data collection. Preliminary results of the image processing provide a qualitative measure of the bed tracer fraction and are included for comparison with the numerical model.

\section{NUMERICAL MODEL}

A simple model for sand fate and the evolution of a tracer field is presented in the following, but these computations are reliant on a suite of supporting predictions. Predictions of the nearshore waves, circulation, and bulk sediment transport, for instance, are required and can be provided by data or a suitable model. The present effort utilizes three models deemed appropriate for nearshore application.

\section{Waves and Circulation}

STWAVE is a steady-state model for nearshore wind-wave evolution and propagation. The steady-state conservation of spectral action balance is solved numerically along backward-traced wave rays using finite difference methods. STWAVE is used routinely on coastal projects involving sediment transport and navigation to estimate the directional wave spectra and to predict wave height, period, and direction. Additionally, STWAVE can be used to predict wave generated radiation stresses in the shoaling and breaking regions. The processes represented in STWAVE include refraction, shoaling, wave-current interaction, wave breaking, and wind wave generation. Input to the model includes bathymetry, offshore spectra, water levels, wind, and currents. Assumptions made in STWAVE include mild bottom slope; steady waves, currents, and winds; refraction and shoaling according to linear theory; depth uniform current; and radiation stresses given according to linear theory. Further details are provided by Smith et al. (2001).

Nearshore circulation acts both to entrain sand and to advect suspended sediments, and an accurate prediction of currents are required as part of this effort. The Adaptive Hydraulics (AdH) Model and the 2D shallow water module presents a suitable two-dimensional finite-element-based horizontal (2DH) hydrodynamic solution of the shallow water equations. In keeping with standard practice of engineering models, a separation of timescales is utilized and the excess wave momentum is approximated by linear radiation stress and applied to the circulation model. The obliquely incident waves in this study results in 


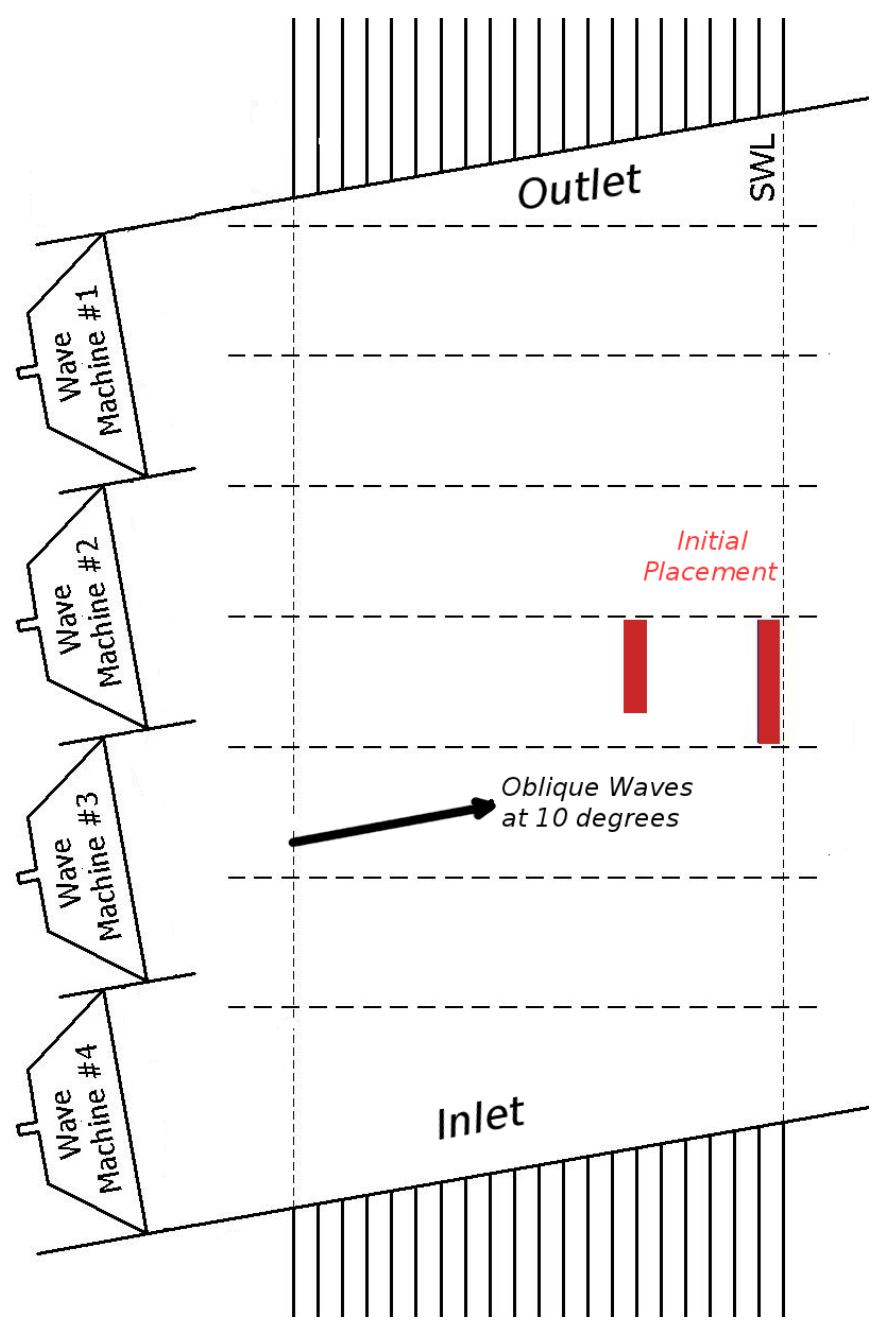

Figure 1. LSTF wave basin and initial dredged mound placement

a free-surface slope in the cross-shore direction and the generation of a steady longshore current. Details of the model formulation and use are available at https://adh.usace.army.mil

\section{Sediment Transport}

Sediment transport rates are predicted based on the computed wave and hydrodynamics from the component models as previously described and utilize the C2SHORE model as introduced in Johnson and Grzegorzewski (2011) and briefly described below. Following the methods of Kobayashi et al. (2009), the instantaneous velocity, $U_{T}$, associated with the random wave is assumed to follow a Gaussian distribution with a zero mean and standard deviation $\sigma_{T}$. The probability density function of a random variable is 
prescribed as

$$
f=\frac{1}{\sqrt{2 \pi}} \exp \left\{\frac{-r^{2}}{2}\right\}
$$

In expression (1), the probabilistic variable $r=U_{T} / \sigma_{T}$. Assuming a relationship between the free-surface oscillation and utilization of the linear long wave theory permits the expression of the standard deviation of wave-orbital velocity in terms of the wave model predictions.

$$
\sigma_{T}=\frac{\sigma_{\eta} c}{h}=\frac{\sqrt{8} H_{r m s} c}{h}
$$

where $\sigma_{\eta}$ is the standard deviation of the free surface position, $H_{r m s}$ is the root-mean square wave height as predicted by STWAVE, $c$ is the linear wave phase speed, and $h$ is the phase-averaged water depth.

Total Bedload Transport

Sediment is assumed to be characterized by a single grain size, $d_{50}$, with a fall velocity $w_{f}$ and sediment specific gravity $s$. The initiation of bed sediment transport is predicted to occur for conditions with an instantaneous bottom shear stress in excess of a critical shear, given in terms of the critical Shields parameter $\psi_{c}$ and expressed

$$
\tau_{c}=\rho g(s-1) d_{50} \psi_{c}
$$

Where $\rho$ is the unit weight of water and $g$ is gravitational acceleration. In the following work, the critical Shields parameter $\psi_{c}$ is assumed constant and equal to 0.05 . The instantaneous bottom shear stress magnitude $\tau_{b}^{\prime}$ is expressed according to the standard quadratic formulation

$$
\tau_{b}^{\prime}=\rho \frac{f_{b}}{2} U_{a}^{2}
$$

where $f_{b}$ is the wave friction factor and $U_{a}$ is the total instantaneous velocity including current and wave orbital components. The expressions above can be combined with the distribution of the wave orbital velocities (1) to compute the probability of bedload sediment movement, $P_{b}$. Recognizing that the probability of sediment movement is equal to the probability of the exceedance of the critical shear leads to the following

$$
P_{b}=\int_{-\infty}^{-\infty} f(r) H\left(\tau_{b}^{\prime}-\tau_{c}\right) d r
$$

where $H$ is Heaviside step function whose value is zero for negative argument and one otherwise. The expression in (5) is integrated numerically to compute $P_{b}$. Previous works detailing the one-dimensional model CSHORE (e.g. Kobayashi et al. 2008, Kobayashi et al. 2009) have assumed currents were small relative to the wave orbital velocities and expressed the bedload as a function of the wave height. In order to permit a more general computation, the previous CSHORE formulations were extended to include current-dominated cases, extending application to cases including overwash and strong tidally generated currents, for instance. As in Kobayashi et al. (2009), the assumption of the classic velocity-cubed bedload model is made when extending the model to include the effect of strong currents. As demonstrated in Johnson and Grzegorzewski (2011), using the total velocity with random waves and currents yields an expression for bedload transport

$$
\left(q_{b x}, q_{b y}\right)=\frac{P_{b}}{g(s-1)}\left[b \sigma_{T}^{3}(\cos \alpha, \sin \alpha)+\left(\frac{f_{b}}{2}\right)^{3 / 2} \gamma\left(F_{U}, F_{V}\right)\right]
$$

where $b$ is is an empirical factor taken as 0.001 in this work, $\alpha$ is the angle of wave propagation, $\gamma \sim 8$ is an empiric current-related factor. The first term in (6) represent wave-driven bedload in the direction 
of wave travel and the second term admits the combined waves and current effects with the velocity moments expressed

$$
\begin{aligned}
F_{U} & =U^{3}+U \sigma_{T}^{2}+U V^{2}+2 \sigma_{T}^{2}(U \cos \alpha+V \sin \alpha) \cos \alpha \\
F_{v} & =V^{3}+V \sigma_{T}^{2}+V U^{2}+2 \sigma_{T}^{2}(U \cos \alpha+V \sin \alpha) \sin \alpha
\end{aligned}
$$

where $U, V$ are the components of the steady current in the $x, y$ direction.

Total Suspended Transport

Following Kobayashi et al. (2009), the degree of sediment suspension is estimated using an empirical expression of the instantaneous turbulent energy dissipation due to bottom friction. It is assumed that the probability of sediment suspension is given by the likelihood that a near-bed turbulent velocity, $k=\left\{f_{b} / 2\right\}^{\frac{1}{3}}\left|U_{a}\right|$, exceeds the sediment fall velocity $w_{f}$. Analogous to the previously shown expression for bedload, the probability of sediment suspension, $P_{s}$ is computed as

$$
P_{s}=\int_{-\infty}^{-\infty} f(r) H\left(k-w_{f}\right) d r
$$

which is numerically integrated. The volume of suspended sediment per unit area, $V_{s}$, is computed as a simple empirical function of the dissipation as introduced in Kobayashi and Johnson (2001):

$$
V_{s}=\bar{c} h=\frac{e_{B} D_{B}+e_{f} D_{f}}{\rho g(s-1) w_{f}} P_{s} \sqrt{1+S_{x}^{2}+S_{y}^{2}}
$$

where $\bar{c}$ is the depth-averaged concentration, $e_{B}$ and $e_{f}$ are empirical suspension efficiencies for the energy dissipation rates $D_{B}$ and $D_{f}$ due to wave breaking and near-bed frictional dissipation respectively, and $S_{x}$ and $S_{y}$ are the bottom slope in the $x$ and $y$ directions. In keeping with Kobayashi et al. (2009), the efficiencies of $e_{B}=0.005$ and $e_{f}=0.01$ are used in this effort. Without regard to the source or details of estimation in the nearshore wave field, the total dissipation rate $D=D_{B}+D_{f}$ is determined from the bulk energy balance, written as

$$
-D=\frac{\partial E_{f x}}{\partial x}+\frac{\partial E_{f y}}{\partial y}
$$

where $E_{f x}, E_{f y}$ are the wave energy flux in the $x, y$ directions respectively. The energy fluxes are computed directly from linear theory and the STWAVE predicted wave characteristics. The time-averaged near-bed frictional dissipation $D_{f}$ uses the previously assumed wave orbital velocity probability density function and quadratic friction

$$
D_{f}=\rho \frac{f_{b}}{2} \overline{U_{a}^{3}}=\rho \frac{f_{b}}{2} \int_{-\infty}^{-\infty}\left\{\left(\bar{U}+r \sigma_{T} \cos \alpha\right)^{2}\left(\bar{V}+r \sigma_{T} \sin \alpha\right)^{2}\right\}^{\frac{3}{2}} f(r) d r .
$$

Suspended sand transport computations depend, in part, on (10) and the depth-averaged currents as predicted by the AdH model. Although the radiation stress is included in the circulation model, the depth-integrated shallow-water equations can not predict the wave-related return current explicitly. However, the return current due to the mass flux of the waves generates the primary erosional off-shore directed transport mechanism within the surf zone. To account for this additional effect, the components of velocity are expressed in a general way with linear wave theory as

$$
\left(U_{R}, V_{R}\right)=-\frac{g H_{r m s}^{2}}{8 c h}(\cos \alpha, \sin \alpha) .
$$


COASTAL ENGINEERING 2012

This relation, in effect, dictates that any wave-generated mass flux is balanced locally by a current below trough level directed in the direction opposite to the wave vector. The accuracy of such an assumption is not fully understood and this contribution must be included cautiously. It is not expected, for instance, to have the effect of a return current in the longshore direction for the case of obliquely incident waves over a cylindrical coast. Nevertheless, it is likely that the mass-flux contribution will be small relative to the pressure driven or radiation stress driven flows developed within the circulation model. For instance, in a moderate wave climate with obliquely incident waves, longshore currents may be $O(1 \mathrm{~m} / \mathrm{s})$ while the wave-generated mass flux distributed over depth is typically $\mathrm{O}(0.1 \mathrm{~m} / \mathrm{s})$. Therefore, violations of the local balance assumption may contribute a small error but will not manifest as unreasonable predictions of suspended sediment transport. Alternatively, the phase-coupling of the time-dependent wave-orbital velocities and sand concentrations can result in a transport aligned with the direction of wave propagation. Based on laboratory measurements detailed in Kobayashi et al. (2005), the magnitude of the wave-related transport was found to scale with the undertow. The proposed expression including the $2 \mathrm{DH}$ current field, return current and wave-related transport can therefore be expressed simply as

$$
q_{s x}=V_{s}\left(U+a U_{R}\right) \quad ; \quad q_{s y}=V_{s}\left(V+a V_{R}\right)
$$

Where $a$ is an empiric factor less then one considering the opposing return current and wave-related transport directions and a value of $a=0.5$ is used throughout this study.

\section{Sand Fate}

The time-evolution of the volume of tracer in two dimensions can be expressed with a depth-integrated conservation statement:

$$
\frac{\partial V^{T}}{\partial t}=-\frac{\partial q_{x}^{T}}{\partial x}-\frac{\partial q_{y}^{T}}{\partial y}
$$

where $V^{T}$ is the total volume of tracer sand per unit area and $q_{x}^{T}, q_{x}^{T}$ are the horizontal tracer transport components. Convectional models solve (15) directly, but this may not be appropriate for surf zone sand fate predictions. Consider, for instance, the case of a simple longshore uniform beach where longshore gradients are negligible and a time-steady equilibrium beach profile. The solution of the depth-integrated expression (15) would then indicate that the tracer field would be time-steady. This, of course, neglects the importance of the vertical structure where the suspended sand is carried offshore and is balanced by a near-bed onshore transport. So where a cross-shore dispersion of tracer sand is expected, the over-simplified single layer model results in unrealistic predictions. In actuality, the variation over the depth leads to an effective diffusivity similar to classic Taylor dispersion. A model is introduced that utilizes a distinction of tracer in the bed and suspended in the water column

$$
\frac{\partial}{\partial t}\left\{V_{b}^{T}+V_{s}^{T}\right\}=-\frac{\partial}{\partial x}\left\{q_{b x}^{T}+q_{s x}^{T}\right\}-\frac{\partial}{\partial y}\left\{q_{b y}^{T}+q_{s y}^{T}\right\}+\mathbf{S}
$$

where subscript $b$ are $s$ denote bedload and suspended load, respectively, and $\mathbf{S}$ is a term accounting for the introduction of tracer from erosion and sequestering through burial. Equation (16) assumes that transport occurs in two distinct modes with a a suspended load within the water column and a bedload part that has a characteristic mixing thickness. This idealized view of two integrated layers is depicted in Figure 2. For an erosive condition, the source and sink term $\mathbf{S}$ accounts for the introduction/sequestration of tracer material from the previously inactive layer. In this case, the source is expressed as

$$
\mathbf{S}=\alpha^{T}(1-p) \frac{\partial z_{b}}{\partial t}
$$


where $\alpha^{T}$ is the fraction of buried inactive sand comprised of tracer material for erosion or in the bed layer for deposition, $p$ is the bed porosity, and $z_{b}$ is the bed position. The two modes can be separated with the introduction of an exchange term $\mathbf{E}$

$$
\begin{aligned}
\frac{\partial}{\partial t}\left\{V_{b}^{T}\right\} & =-\frac{\partial}{\partial x}\left\{q_{b x}^{T}\right\}-\frac{\partial}{\partial y}\left\{q_{b y}^{T}\right\}+\mathbf{E}+\mathbf{S} \\
\frac{\partial}{\partial t}\left\{V_{s}^{T}\right\} & =-\frac{\partial}{\partial x}\left\{q_{s x}^{T}\right\}-\frac{\partial}{\partial y}\left\{q_{s y}^{T}\right\}-\mathbf{E}
\end{aligned}
$$

Recalling that the rates $q_{b}$ and $q_{s}$ are given, the transport of tracer is expressed
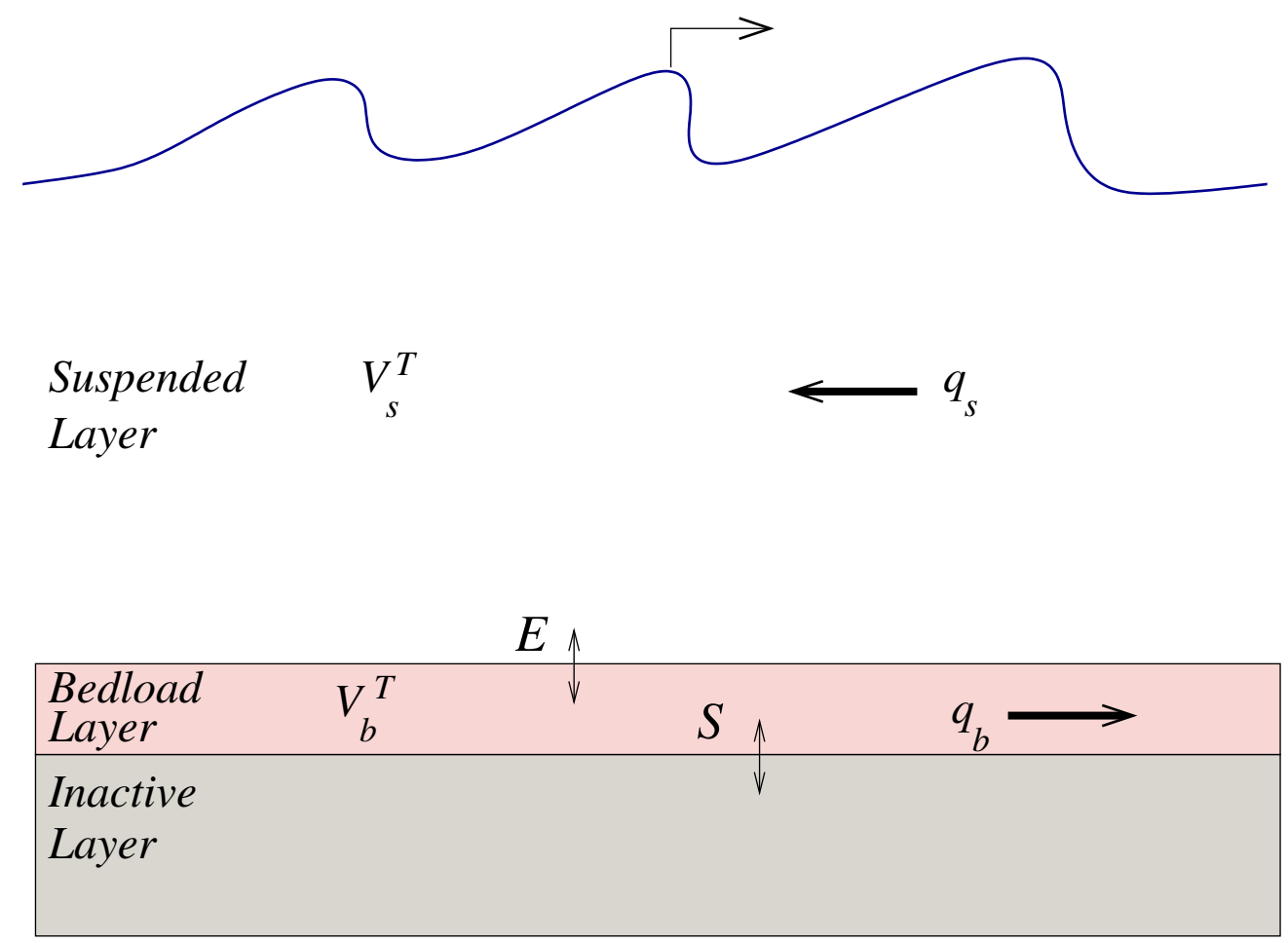

Figure 2. Definition sketch.

$$
\begin{aligned}
& \left(q_{b x}^{T}, q_{b y}^{T}\right)=\alpha_{b}^{T}\left(q_{b x}, q_{b y}\right) \\
& \left(q_{s x}^{T}, q_{s y}^{T}\right)=\alpha_{s}^{T}\left(q_{s x}, q_{s y}\right)
\end{aligned}
$$

where $\alpha_{s}^{T}$ and $\alpha_{b}^{T}$ are the fraction of sand comprised of tracer material in suspension and in the bed respectively. The tracer fraction in suspension and in the bedload layer is given as

$$
\alpha_{s}^{T}=V_{s}^{T} / V_{s} \quad ; \quad \alpha_{b}^{T}=V_{b}^{T} / V_{b}
$$


where the total suspended sand $V_{s}$ is previously introduced and the total volume in the bed $V_{b}$ is developed using the concept of an mixing depth. Defining an active bed layer of thickness $\delta$, the volume is given as $V_{b}=(1-p) \delta$ where $p$ is the bed porosity. Although models are available to predict the active bedload thickness, the present effort makes use of measurements to quantify $\delta$. Most of the sand bed level within the basin remained essentially unchanged for the duration of the experiment. Without net erosion or deposition, the active bed depth was estimated by measuring tracer penetration of core samples collected from positions down-drift of the placement. Although there was variation, a representative value of $1 \mathrm{~cm}$ was used herein. The term $\mathbf{E}$ in (18) represents the exchange of tracer between the bed and the suspended sediment. Conditions of erosion or deposition will generate a vertical tracer flux that is part of the exchange and is analogous to the source expression provided in (17). Even in case of a steady bed, though, the exchange of tracer between the water column and the bed is expected. A rational expression for this mixing is developed with the use of the suspended sand concentration $\bar{c}$ as introduced in (10). For an equilibrium beach, the rate of sand falling out of the water column is balanced by the rate of sand entrainment. A difference in the tracer concentrations in suspension and in the bed, then, will result in a net transport. Thus the exchange rate is shown in 2 and is comprised of two parts:

$$
\mathbf{E}=\alpha(1-p) \frac{\partial z_{b}}{\partial t}+\bar{c} w_{f}\left(\alpha_{b}-\alpha_{s}\right)
$$

where $\alpha$ is the fraction in the bed/suspension for erosion/deposition, and $\bar{c} w_{f}$ is an estimate of the equilibrium total sand exchange [e.g. Nielsen 1992]. Note that the second term of $E$ functions as a gradient diffusion mechanism, acting to bring equilibrium to the tracer fraction in the bed and in suspension.

The set of coupled equations in (18) are solved using a first-order explicit Euler finite difference scheme. To avoid excessive numerical diffusion, the time increment is determined according to the linear stability criteria. The time step $\Delta t$ is limited by the suspended transport mode and is given according to

$$
\Delta t=C n \frac{\Delta x}{u_{\max }}
$$

where $C n$ is the Courant number and the maximum sediment velocity $u_{\max }$ is given by $\max \left\{\left|q_{s y}\right| / V s,\left|q_{s x}\right| / V s\right\}$. Although a Courant number of 1.0 is theoretically stable, a value of 0.5 was used for greater stability and minimal numerical diffusion.

\section{RESULTS}

Modeling sediment transport is a formidable challenge, in part because reliable predictions are contingent on the accurate representation of the underlying hydrodynamics and morphology. In a nearshore domain, for instance, a proper assessment of the tracer mixing and transport model is dependent on accurate prediction of bed levels, waves, currents, etc. To reduce ambiguity in the present effort, use is made of calibrated models as well as measured data. A representation of the bottom position change, for example, is necessary, and the collected bathymetry data are used. The tracer placement is relatively small, standing proud of the longshore uniform profile by less than $7 \mathrm{~cm}$. In both Test 1 and Test 2, the mound was quickly removed in the energetic surf zone environment. Figure 3 shows an example of the bed change following ten minutes of waves and currents for Test 2 . It is clear that the mound was essentially eroded away with $7 \mathrm{~cm}$ of lowering while the remainder of the basin shows little change. The results of Test 1 are not show here for brevity, but the placement is removed more slowly, but the majority of the feature was eroded within 20 minutes.

The relatively small placement is only a minor perturbation to an otherwise longshore uniform domain. The modeled hydrodynamic predictions, therefore, are provided for comparison to measured data along a single cross-shore transect, and the accuracy of the provided comparisons are representative 


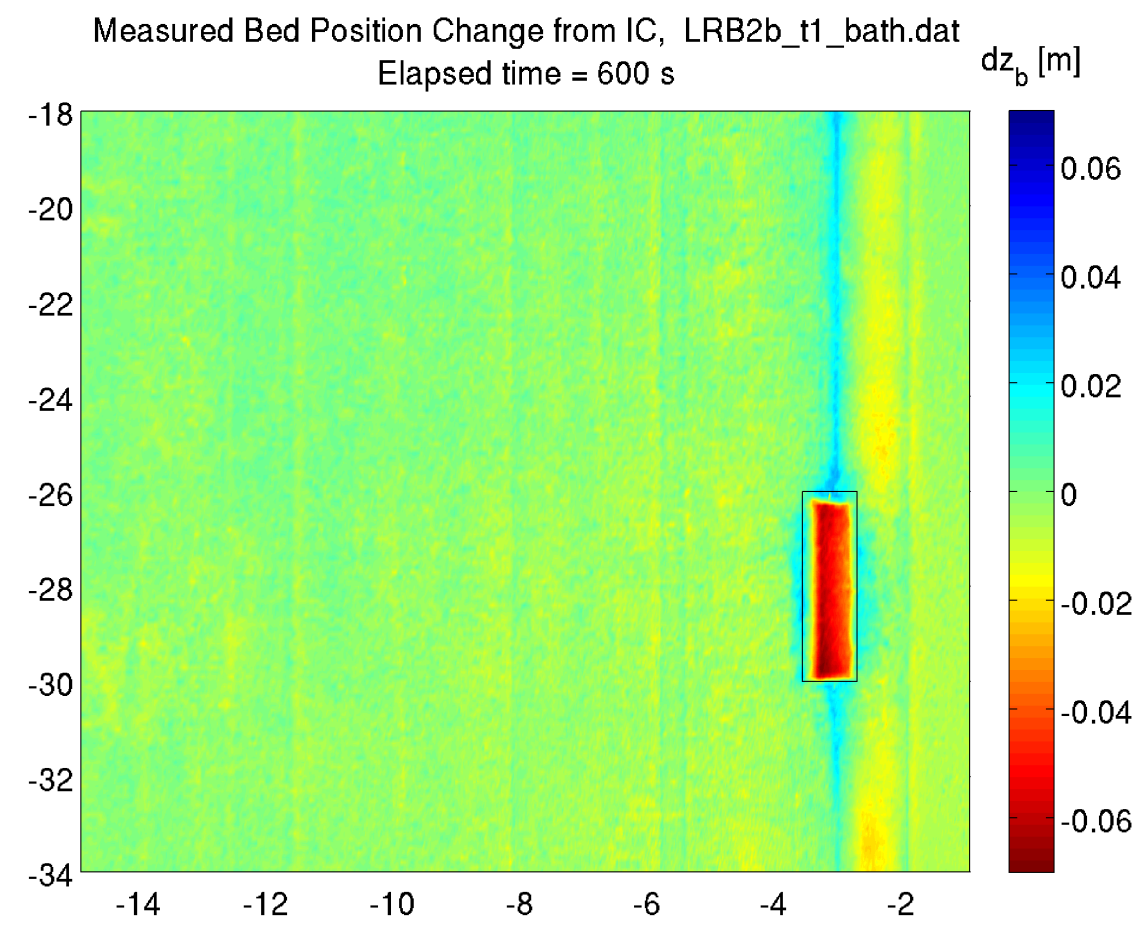

Figure 3. Bed level change after 10 minutes

of the results in general. Figure 4 shows the measured wave heights and the predictions of the STWAVE model. The intention of this effort was to verify the tracer model, and the model was simply calibrated to provide accurate wave heights. The cross-shore decay in wave energy is reasonably well predicted with the greatest discrepancy at the most shallow gauge, in $8 \mathrm{~cm}$ of water, where the measured root-mean-square wave height is $10 \%$ greater than the model prediction. The obliquely incident breaking wave field drives a longshore current, and the depth-averaged velocity is shown in Figure 4. The circulation model makes use of the quadratic bottom shear stress formulation with an empirical friction coefficient. Again, to provide a accurate hydrodynamic field to the tracer fate model, the coefficient is selected for the best fit to measured data. The agreement is reasonable over most of the surf zone. In this laboratory study, the primary offshore-directed tracer transport is due to the return current. The wave-generated undertow is not directly predicted by the AdH model, though, and use is made of the previously introduced expression (13). The depth-variation of the cross-shore currents was not measured, but the data collected at a single vertical position measured one third of the the depth from the bed are provided in panel 3 of Figure 4 along with the prediction. The expression for undertow may be over-simplified without including the mass flux of a wave roller, but the predictions compare well with the measured data.

The sand fate model is initiated with an active bed layer and buried layer that are $100 \%$ tracer sand within the footprint of the placement site as shown in Figure 1. The first row of plots in Figure 5 show a 
representation of the tracer fraction at the bed surface for the initial conditions for Test 1 . The measured data are based on image processing as previously explained, and the presented model results are the tracer concentration within the bed layer. Subsequent row of plots provide snapshots of tracer fraction after waves and currents are run for 10 and 20 minutes, respectively. The measured data indicate transport of the tracer that is directed downstream with the longshore current and offshore due to the undertow. Movement onshore, attributable to wave-related transport, is limited with a moderate migration of the tracer onshore indicated at $t=20$ minutes. Likewise, the model predicts an onshore-directed bedload layer for this essentially longshore uniform case with small cross-shore currents as explicitly formulated in (6). To a greater degree, the tracer in suspension is advected offshore and downstream, and the extent of spread increases with time. Although results of the image analysis are imprecise, the time-evolution of the model and data surface layers are qualitatively similar. In general, the model results in a greater degree of dispersion when compared with the measurements, but the direction of migration is well predicted.

The results of the onshore placement in Test 2, provided in Figure 6, differ in comparison to the first test. In keeping with the large measured currents in the shallow water seen in Figure 4, the image analysis indicates dominant sand advection in the longshore direction. Also, the onshore movement of tracer is more pronounced and is slightly larger in magnitude than the offshore transport. The modeled results indicate tracer dispersion in the cross-shore that compares well, in general, to the data. The rate of migration in the longshore direction is smaller in the model, perhaps in part due to the under-prediction of the current magnitude.

\section{CONCLUSIONS}

Following decades of development, models for waves transformation and surf zone circulation abound. And despite the difficulty in predicting nearshore morphology, methods are now available that provide a representation of the physical processes of sand transport. While this $2 \mathrm{DH}$ class of models is stable and efficient, no physically justifiable mechanism for predicting sand fate and dispersion within the surf zone is available. An alternative approach is developed herein, where the bed layer and suspended layer can have arbitrary transport directions and are solved as a set of coupled equations. To validate the conceptual model, tests were conducted in a laboratory basin with obliquely incident waves and a longshore current. Sand was dyed to provide distinguishing tracer characteristics, and the material was placed in two locations within the surf zone. The model and the data both indicated a moderate movement of the tracer sand in the deeper placement with a slight migration downstream and offshore. The shallow placement was characterized by a greater cross-shore dispersion and dramatic longshore transport. The model results for the second test were in general agreement with data but exhibit an under-prediction of longshore migration .

\section{ACKNOWLEDGMENTS}

The Chief of Engineers, US Army Corps of Engineers, is acknowledged for granting permission to publish this work.

\section{REFERENCES}

Dalrymple, R.A. 1992. "Prediction of storm/normal beach profiles," Journal of Waterway, Port, Coastal and Ocean Engineering, ASCE, 118(2),193-200.

Johnson, B., and Grzegorzewski, A.S. 2011. "Modeling Nearshore Morphologic Evolution of Ship Island During Hurricane Katrina,” Proceedings of Coastal Sediments 2011, World Scientific, 1797-1810.

Hamilton, D.G., B.A. Ebersole, E.R. Smith, and P. Wang. 2000. "Development of a large-scale laboratory facility for sediment transport research," Technical Report, ERDC/CHL TR-01-22, Vicksburg, MS.

Kobayashi, N., and B.D. Johnson. 2001. "Sand suspension, storage, advection and settling in surf and swash zones," Journal of Geophysical Research, 106(C5) 9363-9376. 
Kobayashi, N., Payo, A., and Schmied, L. 2008. "Cross-Shore Suspended Sand and Bedload Transport on Beaches," Journal of Geophysical Research, 113, C07001, doi:10.1029/2007JC004203

Kobayashi, N., Payo, A., and Johnson, B.D. 2009."Suspended sand and bedload transport on beaches," Handbook of Coastal and Ocean Engineering, World Scientific. Chapter 28, 807-823.

Kobayashi, N., Zhao, H. and Tega, Y. 2005. "Suspended Sand Transport in Surf Zones", Journal of Geophysical Research, 110, C12009, doi:10.1029/2004JC002853.

Nielsen, P., 1992. Coastal Bottom Boundary Layers and Sediment Transport. World Sci., River Edge, NJ.

Smith, J.M., Sherlock, A.R., and Resio, D.T. 2001. "STWAVE: steady-state spectral wave model user's guide for STWAVE." Special Report ERDC/CHL-01-01 US Army Engineer Research and Development Center, Vicksburg, MS. 

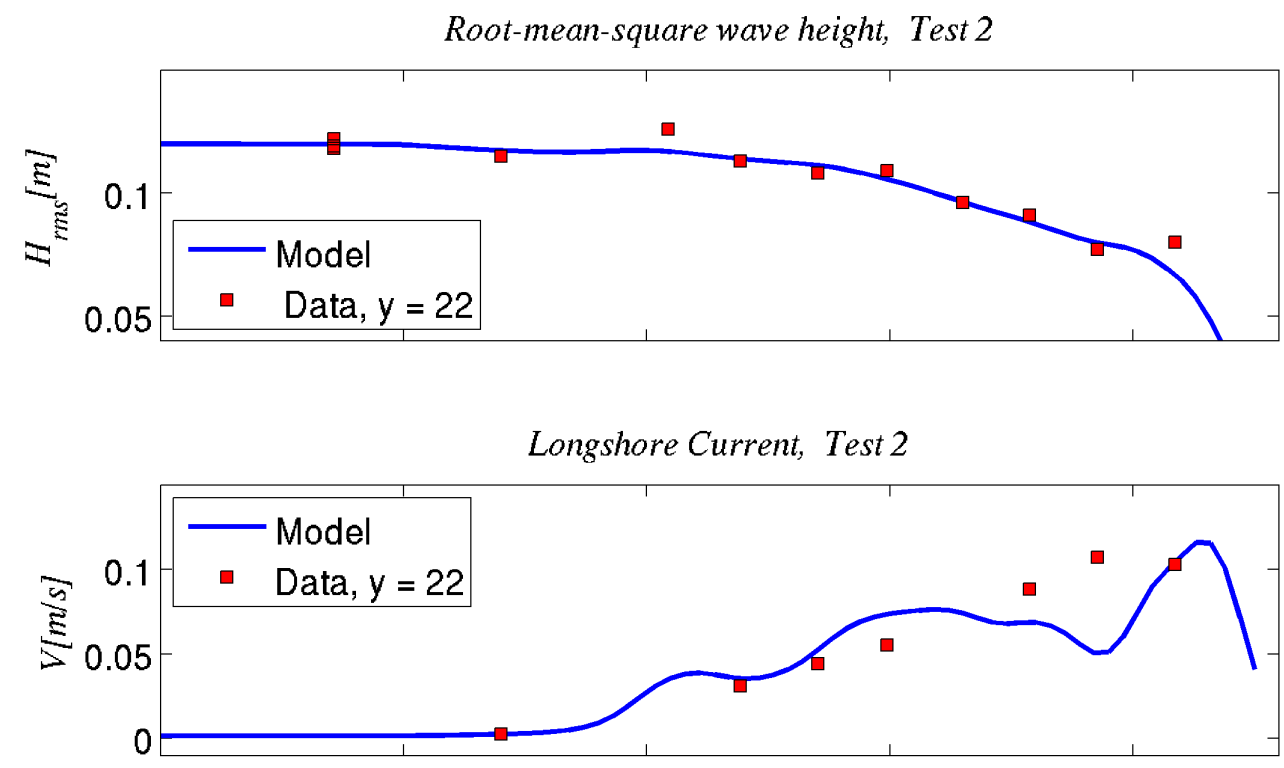

Return Current, Test 2
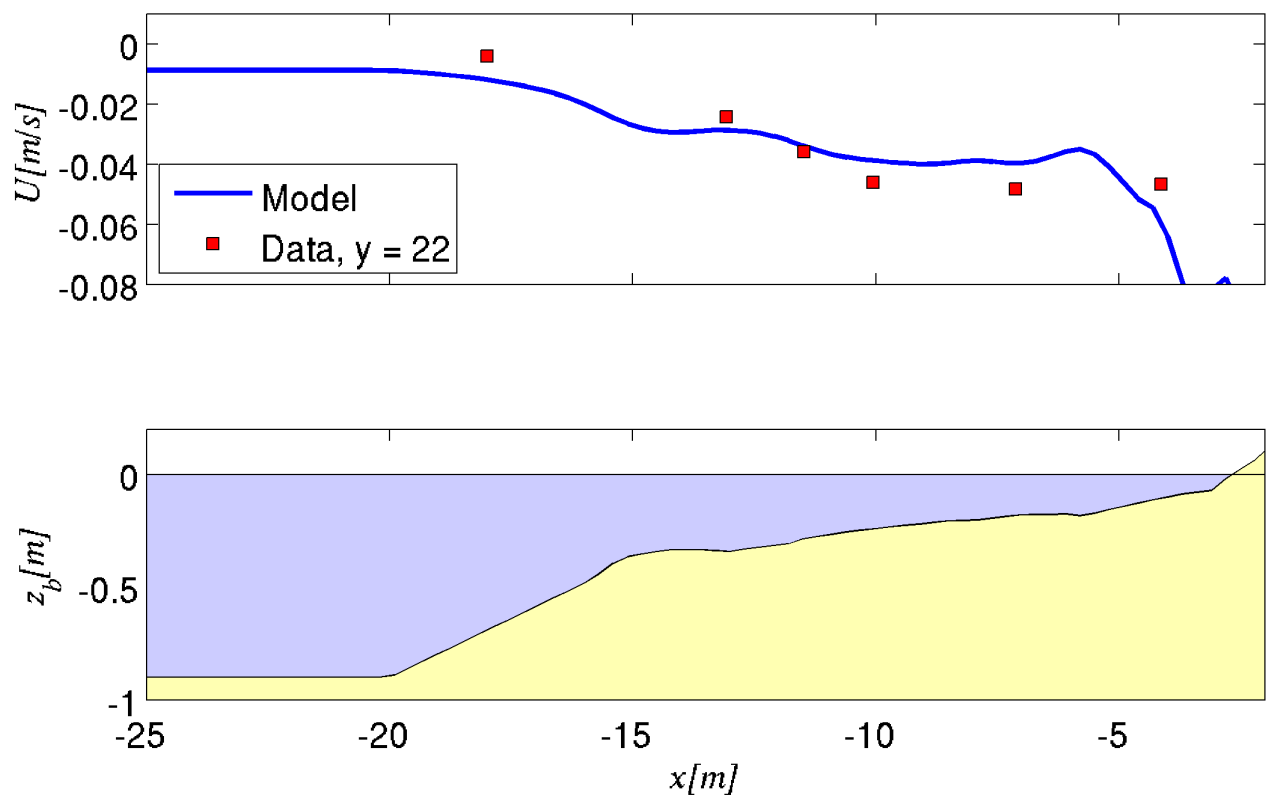

Figure 4. Model and data waves and currents. 

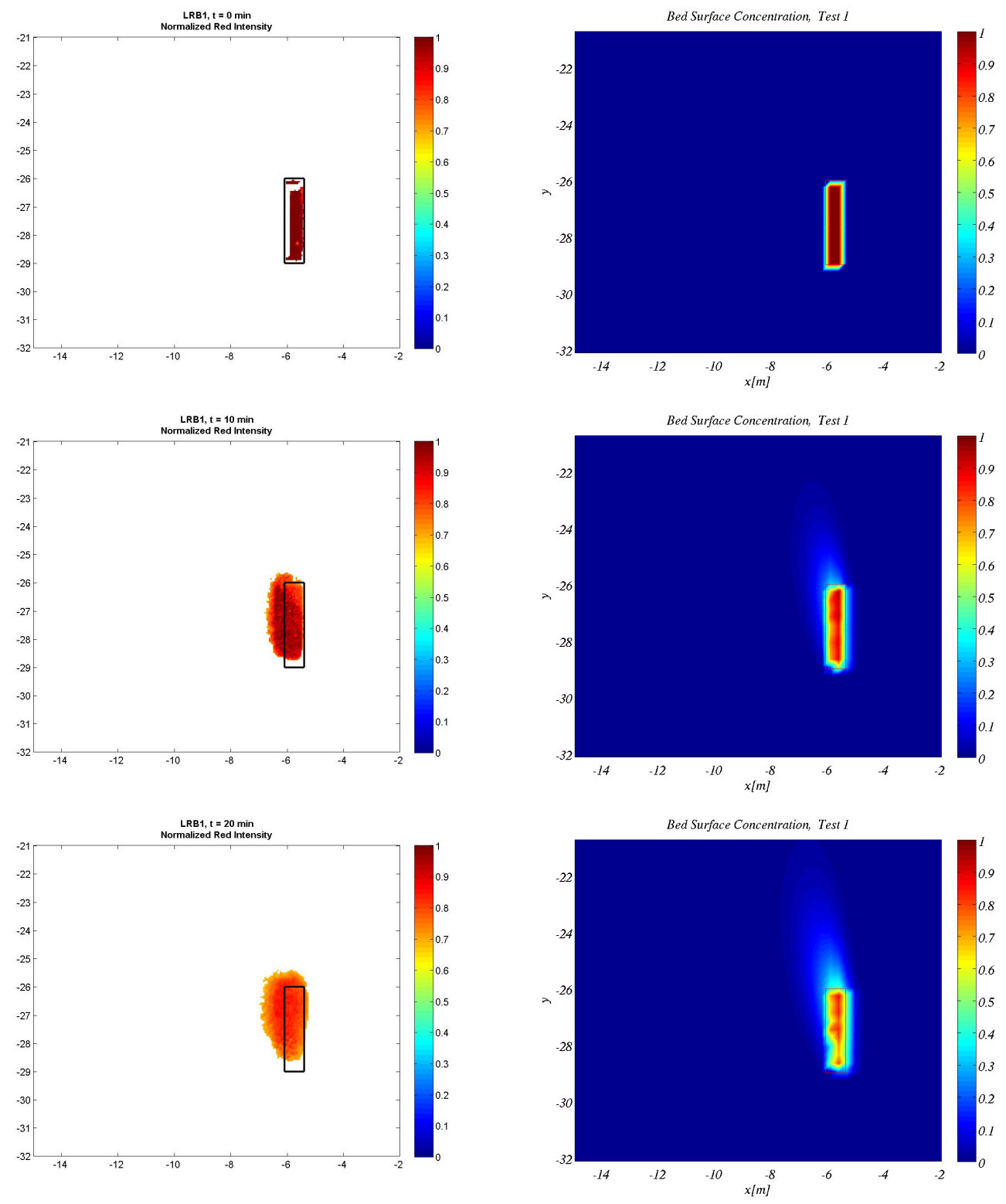

Figure 5. Bed surface tracer concentrations for Test 1. 

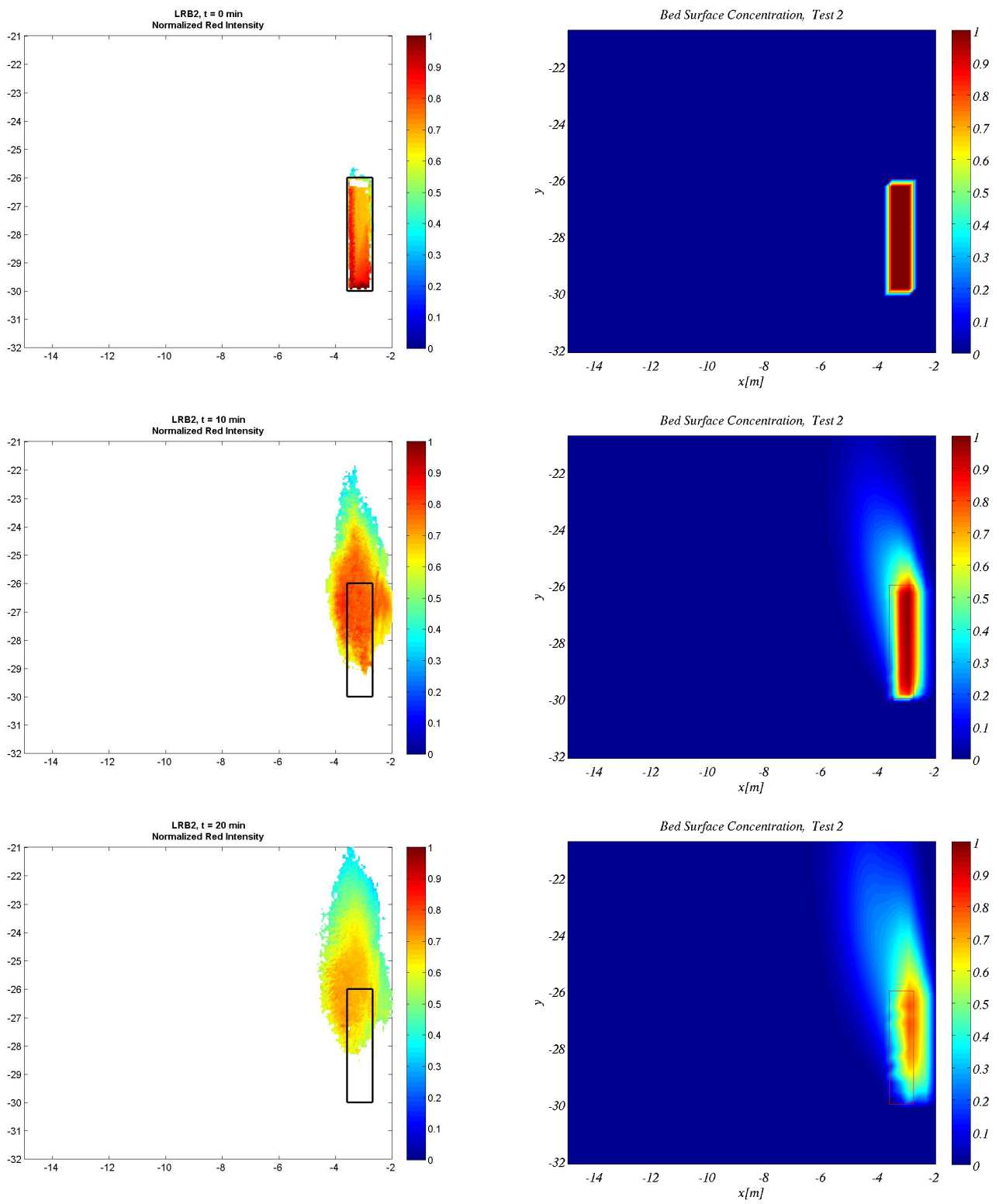

Figure 6. Bed surface tracer concentrations for Test 2. 